\title{
Direct Evidence of Two Equilibration Mechanisms in Glassy Polymers
}

\author{
Daniele Cangialosi, ${ }^{1}$ Virginie M. Boucher, ${ }^{1}$ Angel Alegría, ${ }^{1,2}$ and Juan Colmenero ${ }^{1,2,3}$ \\ ${ }^{1}$ Centro de Física de Materiales (CSIC-UPV/EHU), Paseo Manuel de Lardizabal 5, 20018 San Sebastián, Spain \\ ${ }^{2}$ Departamento de Física de Materiales, Universidad del País Vasco (UPV/EHU), Apartado 1072, 20080 San Sebastián, Spain \\ ${ }^{3}$ Donostia International Physics Center, Paseo Manuel de Lardizabal 4, 20018 San Sebastián, Spain
}

(Received 4 June 2013; published 27 August 2013)

\begin{abstract}
We investigated the kinetics of enthalpy recovery of several glass-forming polymers at temperatures significantly below the glass transition temperature $\left(T_{g}\right)$ and for aging times up to one year. We find a double-step recovery at relatively low aging temperatures for the longest investigated aging times. The enthalpy recovered after the two-step decay approximately equals that expected by extrapolation from the melt. The two-step enthalpy recovery indicates the presence of two time scales for glass equilibration. The equilibration time of the first recovery step exhibits relatively weak temperature dependence, whereas that of the second step possesses pronounced temperature dependence, compatible with the VogelFulcher-Tammann behavior. These results, while leaving open the question of the divergence of the relaxation time and that of a thermodynamic singularity at a finite temperature, reveal a complex scenario of glassy dynamics.
\end{abstract}

DOI: 10.1103/PhysRevLett.111.095701

PACS numbers: 64.70.pj, 65.60.+a

Nowadays, it is well known that glassy materials can be obtained by cooling down a liquid if crystallization is avoided [1]. The process of formation of a glass is called the glass transition and is kinetic in nature. Hence, the temperature associated with this process $\left(T_{g}\right)$ depends on the cooling rate of the experiment, since the time needed to equilibrate the liquid, the equilibration time $\tau_{\text {eq }}$, is temperature dependent. As the temperature crosses the region around $T_{g}, \tau_{\mathrm{eq}}$ becomes comparable to the time scale of the experiment related to the inverse of the cooling rate $q$, and thereby the system becomes a nonequilibrium glass. Below $T_{g}$, the glass slowly evolves with time toward equilibrium, a phenomenon known as physical aging [2,3]. Conventionally and in the rest of the Letter, the $T_{g}$ is taken as that corresponding to standard calorimetric experiments, that is, those performed at several kelvin per minute corresponding to an observation time scale of several seconds.

Several studies have univocally related $\tau_{\text {eq }}$ to the typical relaxation time $\tau_{\text {mol }}$ of molecular spontaneous fluctuations associated to the $\alpha$ process [4-7], suggesting that this is the only process relevant for the glass transition. $\tau_{\mathrm{mol}}$ is generally described by the empirical Vogel-Fulcher-Tammann (VFT) equation [8-10]

$$
\tau_{\text {mol }}=\tau_{0} \exp \left(\frac{B}{T-T_{0}}\right),
$$

where $\tau_{0}$ is a prefactor, and $T_{0}$ and $B$ are the Vogel temperature and activation energy, respectively. The VFT law insinuates divergence of the relaxation time at $T_{0}$.

A connection of dynamics with thermodynamics has been inferred in the past. In particular, analysis of thermodynamic data on a wide range of glass formers indicates that extrapolation of the behavior normally observed at relatively high temperature produces a singularity at a finite temperature $T_{K}$, where the entropy of the liquid would equal that of the corresponding crystal [11]. If the contribution of the entropy exclusively related to the $\alpha$ process is considered [12], $T_{0} \approx T_{K}$ is generally encountered [13].

The previous description is based on the common view that a single time scale of equilibration, associated with a single $T_{g}$, is present in glass-forming systems. However, there exist at least two studies, one on a siloxane based molecular glass former [14] and the other on thin polystrene (PS) films [15], suggesting the presence of two equilibration time scales, since two jumps in second order thermodynamic properties were detected in those studies. These would be a manifestation of the existence of two equilibration mechanisms with rather different time scales.

The vast majority of experiments are performed on typical observation time scales shorter than several seconds, that is, above the conventional $T_{g}$. However, in recent years, the previous issues have been tackled by performing experiments in the so-called aging regime as a way to explore the ultraslow molecular dynamics range. In particular, either the kinetics of recovery of thermodynamic (or related) properties or the evolution of the relaxation time during physical aging were monitored on time scales considerably larger than several seconds. Several of these studies suggest that deviations from the VFT behavior of $\tau_{\text {eq }}$ to a milder temperature dependence occur at large time scales of observation [16-24]. This finding generated (or has been generated by) intense theoretical activity, accounting for this kind of behavior [25-28]. Conversely, other approaches rather predict divergence of the relaxation time in line with the empirical VFT law [29,30]. At the same time, several studies in glassy polymers indicated that after prolonged aging at temperatures significantly 
below $T_{g}$, a plateau in the enthalpy is found with enthalpy values considerably larger than those corresponding to the thermodynamic state extrapolated from above $T_{g}$ [22,31-35]. An exception is represented by a recent work where a single-step decay to the plateau expected from extrapolation from above $T_{g}$ is found for PS at $358 \mathrm{~K}$ [36]. However, it is worth remarking that in the latter case, differently from Refs. [22,32,35], where a plateau with partial enthalpy recovery is found for PS, samples with a wide distribution of molecular weights are employed. The presence of an intermediate plateau is likely also the case of recent experiments by Welch et al. [37] on an inorganic glass, who showed that a plateau in a volume related recovery function is achieved within one year at a temperature as low as $600 \mathrm{~K}$ below $T_{g}$. Furthermore, experiments within the so-called asymmetry of approach demonstrated that the obtained plateau corresponds to a relative minimum in the energy landscape [22]. These results leave open the question on whether additional decays in the enthalpy recovery would be observed if longer aging times were considered, which implies the presence of distinct mechanisms of equilibration.

In this work, we study the enthalpy recovery of three glassy polymers, where the value of the enthalpy at the plateau was found to be significantly different from that expected by extrapolation from the melt [22]. These polymers are high- and low-molecular-weight polystyrene (for PS85k, $M_{n}=85 \mathrm{~kg} / \mathrm{mol}, T_{g}=375 \mathrm{~K}$; for PS7k, $M_{n}=7 \mathrm{~kg} / \mathrm{mol}, T_{g}=363 \mathrm{~K}$ ) and polycarbonate (for PC36k, $M_{n}=36 \mathrm{~kg} / \mathrm{mol}, T_{g}=419 \mathrm{~K}$ ) at temperatures significantly below $T_{g}$ and for aging times as long as about one year. In doing so, we extend previous enthalpy recovery studies [22] to rarely explored aging times, in particular, those larger than those needed to reach the previously mentioned plateau. We show that further enthalpy recovery occurs after large aging times and, in those cases where a second plateau can be reached, the final thermodynamic state approximately corresponds to that of the extrapolated melt. This two-step enthalpy recovery defines two equilibration time scales. The faster one exhibits activation energy significantly smaller than that of the $\alpha$ process at $T_{g}$, whereas the slower time scale is compatible with VFT behavior.

The enthalpy recovery has been investigated by differential scanning calorimetry (DSC), employing a DSC-Q2000 from TA Instruments. This technique delivers the temperature dependent specific heat, which at constant pressure equals the temperature derivative of the enthalpy. All enthalpy recovery experiments began with a heating ramp to a temperature equal to or larger than $T_{g}+30 \mathrm{~K}$, kept for $5 \mathrm{~min}$, to erase the material's previous thermal history. Samples were subsequently cooled down at $20 \mathrm{~K} \mathrm{~min}^{-1}$ at a temperature considerably lower than the aging temperature $T_{a}$, followed by stabilization at $T_{a}$ and aged in the calorimeter for times from several minutes to
$48 \mathrm{~h}$ before being cooled down to a temperature considerably lower than $T_{a}$, at a cooling rate of $20 \mathrm{~K} \mathrm{~min}^{-1}$, prior to reheating at $10 \mathrm{~K} \mathrm{~min}^{-1}$ for data collection (aged scan). For the measurements of the enthalpy recovery at longer aging times $\left(t_{a}>48 \mathrm{~h}\right)$, the annealing of the samples was carried out in an external vacuum oven, at $T_{a}$, after the erasing of the thermal history and cooling of the samples in the DSC. After aging, the samples were cooled down again and DSC thermograms recorded. Second scans were run immediately after a new cooling at $20 \mathrm{~K} \mathrm{~min}^{-1}$. The complete thermal procedure applied to the samples for the structural recovery study has been schematized in a previous work [38]. As a general rule, the amount of recovered enthalpy of a glass for a period of time $t_{a}$ at a given temperature $T_{a}$ was evaluated by integration of the difference between thermograms of aged and unaged samples, according to the relation [4]

$$
\Delta H\left(T_{a}, t_{a}\right)=\int_{T_{x}}^{T_{y}}\left[C_{p}^{a}(T)-C_{p}^{u}(T)\right] d T,
$$

where $C_{p}^{a}(T)$ and $C_{p}^{u}(T)$ are, respectively, the specific heat of the aged and unaged samples, and $T_{x}$ and $T_{y}$ are, respectively, temperatures well below and above the calorimetric $T_{g}$ [4].

Figure 1 shows the temperature dependence of the specific heat at some selected aging times-chosen in a way that $\Delta \log t_{a}$ between two consecutive scans is approximately constant-for PS85k at $363 \mathrm{~K}$. As is customary in enthalpy recovery experiments, physical aging manifests with the development of an endothermic overshoot, whose magnitude increases with aging time. However, a first qualitative observation of the figure indicates that the growth rate of the overshoot is nonmonotonic. In particular, at aging times between half and several days, the development of the overshoot appears to be considerably slower in comparison to shorter and larger aging times.

The latter experimental result can be quantitatively explored by calculating the recovered enthalpy employing Eq. (2). The decay in the enthalpy during physical aging is

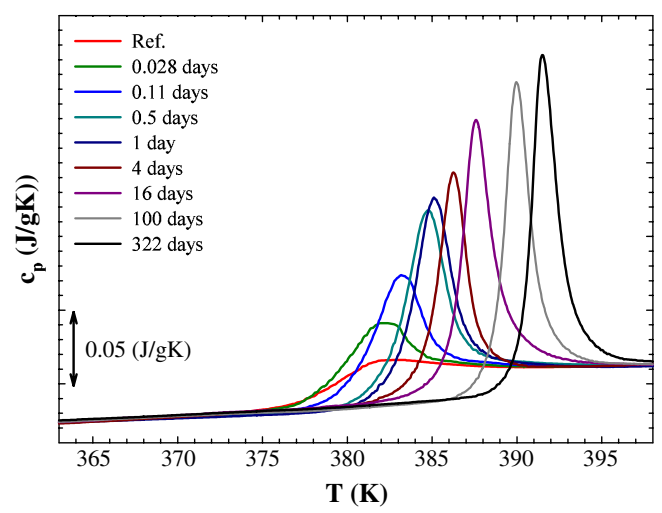

FIG. 1 (color online). Specific heat versus temperature as obtained by DSC for PS85k at $363 \mathrm{~K}$ at different aging times. 
shown in Figs. 2(a)-2(c) for all investigated polymers. This is displayed as the difference between the total recoverable enthalpy and the enthalpy recovered at a given aging time $\Delta H(t)$. The former is calculated by extrapolating the enthalpy of the equilibrium melt to the aging temperature as $\Delta H_{\mathrm{tot}}=\int_{T_{a}}^{T_{g}} \Delta C_{p} d T$, where $\Delta C_{p}$ is the difference between the melt and glass specific heat and is taken from Ref. [22]. The behavior of the enthalpy recovery exhibits the following main characteristics: (i) in the proximity of $T_{g}$, the recovery exhibits the standard single-step decay; (ii) at temperatures significantly below $T_{g}$, enthalpy recovery proceeds via a two-step process; and (iii) in all cases where the final plateau is achieved, in a good approximation, the recovered enthalpy corresponds to that extrapolated from the melt. The latter aspect is evidenced in the inset of Fig. 2(d) where the enthalpy recovered at each plateau is shown as a function of temperature together with the extrapolated melt line for PS85k as a showcase.

The most relevant result in the context of the present study is the presence of a two-step decay in enthalpy recovery, schematically depicted in Fig. 2(d). This is also found in PS7k, that is, a polymer with molecular weight significantly below that between entanglements (17 kg/mol for PS). While some previous studies on different kinds of inorganic glasses $[39,40]$ as well as two works on the long term volume recovery of polycarbonate (PC) at room temperature [41,42] provide some indications on such behavior, to our knowledge, this is the first systematic study providing unambiguous evidence of a two-step decay for different polymers at several temperatures.

The previous results imply that enthalpy recovery in glassy polymers exhibits two time scales of equilibration. A way to obtain information on the temperature behavior of these time scales is to extract the time of equilibration to each plateau of the recovery. This is analogous to the method, employed in previous works [18,22], based on the determination of the shift factor obtained by aging time-temperature superposition of enthalpy recovery data close to the plateau. The time of equilibration, obtained through the geometric construction shown in Fig. 2(a) as a showcase, for all investigated polymers, is shown in Fig. 3 as a function of the inverse temperature. A splitting (merging) scenario is evidenced in the figure resulting from the presence of a single equilibration mechanism at relatively high temperatures and a double decay recovery at lower temperatures. As can be observed, the time scale for equilibration at the first plateau exhibits relatively low activation energy seemingly decreasing with temperature and of the order of a few hundreds of $\mathrm{kJ} \mathrm{mol}^{-1}$. The temperature dependence of the equilibration time of the first plateau was examined in a previous work
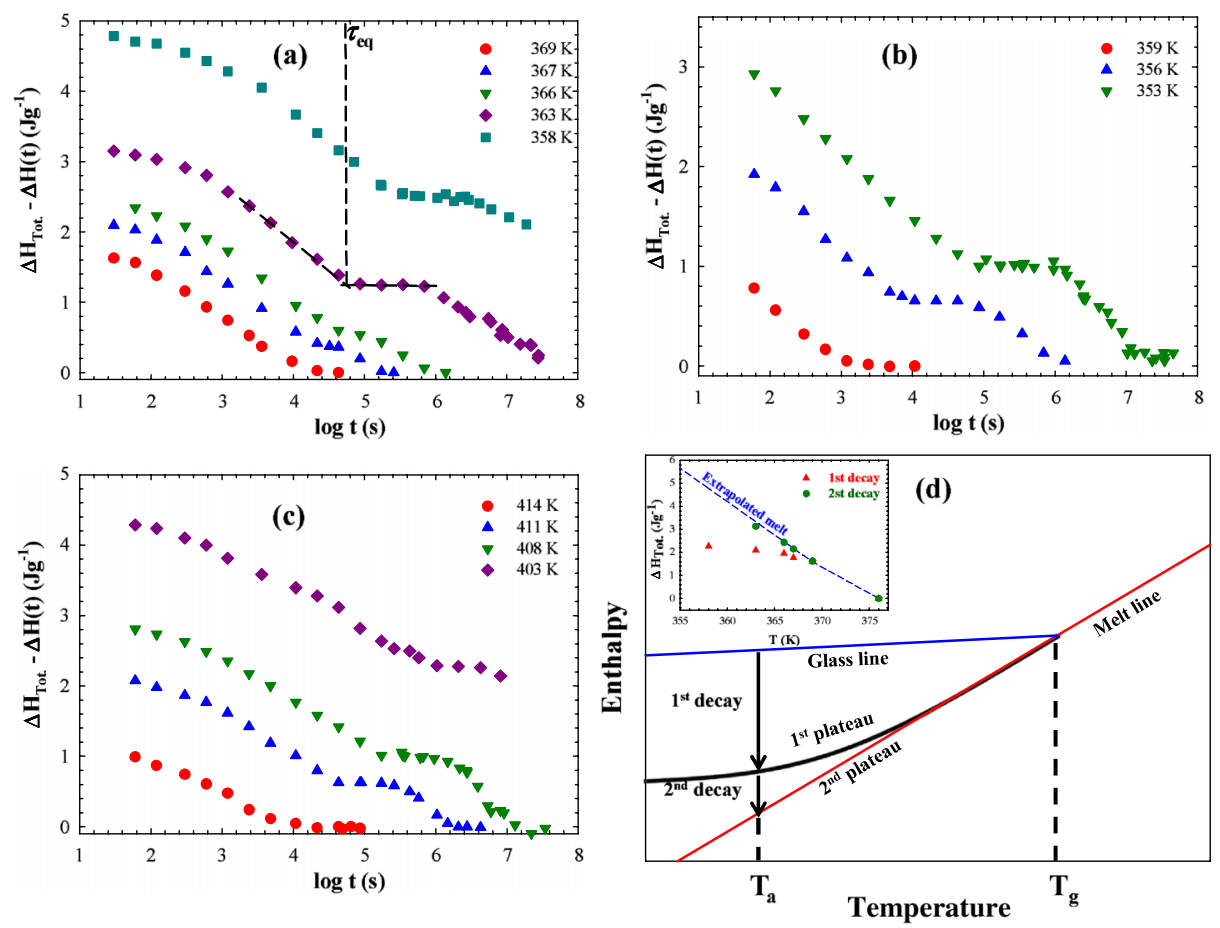

FIG. 2 (color online). Time evolution of the recovered enthalpy at the indicated temperatures for (a) PS85k, (b) PS7k, and (c) PC36k. The experimental uncertainty in the amount of recovered enthalpy is $\pm 0.05 \mathrm{Jg}^{-1}$. The dashed lines in (a) are the logarithmic fits to enthalpy recovery data in the time interval of maximum variation and at the plateau. The intersection of the two fits provides welldefined values of the equilibration time. The mean standard error is $\Delta \log \tau_{\mathrm{eq}}= \pm 0.2$. (d) Schematically shown is the temperature behavior of the enthalpy at each (relative) minimum in the energy landscape. The inset of (d) shows experimental points for PS85k as a showcase of the enthalpy recovered at each plateau and the extrapolation of the enthalpy from the melt. 


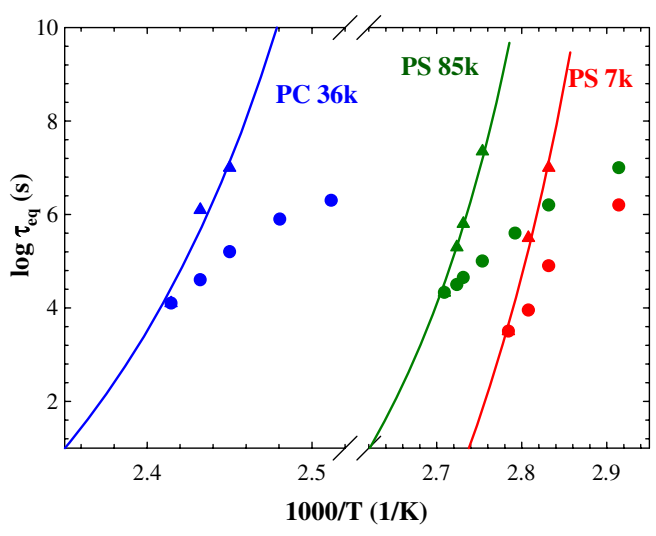

FIG. 3 (color online). Logarithm of equilibration times corresponding to the first (circles) and the second (triangles) plateaus as a function of the inverse temperature obtained from enthalpy recovery data and derived from the temperature dependence of the $\alpha$ relaxation according to the VFT equation (lines). The error bar in the values of the equilibration times is in all cases $\Delta \log \tau_{\mathrm{eq}}= \pm 0.2$. as estimated from the construction of Fig. 2(a). The VFT parameters are $\log \tau_{0}=-10.17 \mathrm{~s}$, $B=1325 \mathrm{~K}$, and $T_{0}=330 \mathrm{~K}$ for PS85k; $\log \tau_{0}=-16.7 \mathrm{~s}$, $B=1930 \mathrm{~K}$, and $T_{0}=318 \mathrm{~K}$ for PS7k; and $\log \tau_{0}=$ $-11.41 \mathrm{~s}, B=1500 \mathrm{~K}$, and $T_{0}=373 \mathrm{~K}$ for PC36k.

[22]. In the case of the equilibration time of the second plateau, a considerably more marked temperature dependence can be observed. Interestingly, the temperature dependence of these equilibration times is adequately caught by the VFT law. This is shown in Fig. 3 where the continuous lines are the fits of the VFT law to the longest equilibration time. Here, the parameters $B$ and $T_{0}$ of the VFT equation have been fixed to those obtained by dielectric relaxation spectroscopy [22]. Hence, an important conclusion of this analysis is that the equilibration time to the second plateau exhibits the same temperature dependence as that of $\tau_{\mathrm{mol}}$ obtained by independent characterizations.

Enthalpy recovery results, showing the existence of double decay, indicate a complex behavior of both thermodynamics and dynamics. Regarding the first decay, an intuitive explanation, based on the relatively low activation energy, is that secondary relaxations are responsible for such decay. However, this explanation would be at odds with the separation in time scales of the first decay of the two investigated PSs (see Fig. 3). It is in fact well established that the time scale of secondary relaxations does not depend on the molecular weight. Nonetheless, it is noteworthy that the separation of time scales of the two PSs becomes smaller when decreasing the temperature. This observation leaves open the possibility that secondary relaxations are responsible for the first decay. Alternative molecular mechanisms, bearing no relation with secondary relaxation processes, could also be invoked to explain the first decay in the enthalpy. In such a case, it is worth mentioning that several experiments in thin PS films conducted in a sub- $T_{g}$ temperature range show the presence of weakly activated molecular relaxation processes $[43,44]$.

The temperature dependence of the equilibration time to the second decay is adequately described by the same VFT law employed to fit dielectric relaxation data above $T_{g}$ [22]. This result indicates that the full recovery of the enthalpy must be driven by the polymer $\alpha$ process. Importantly, in the temperature interval explored in the present work, where full equilibration of the glass is allowed in a time scale of one year, no clear deviations from the VFT law are observed. This result apparently contrasts with those reporting significant deviations $[17,18]$. We speculate that the deviations observed in those studies may be related to the fact that physical aging in the first decay regime is explored. This interpretation is corroborated by the observation that the temperature dependence of the shift factor obtained from stress relaxation experiments in PC [17] appears to match the temperature dependence of the first decay of the present study. Our results leave open the question of the thermodynamic and dynamic singularity at a finite temperature. Recent results on amber glass aged for millions of years suggest that glasses significantly lower in the landscape than those of the present study exhibit deviations from the VFT law [45].

An important implication of the presence of two time scales of equilibration in the enthalpy is that a glass former cooled at sufficiently low rates would exhibit two jumps in the heat capacity. In the case of the glass-forming polymers of the present study, feasible cooling rates $\left(>10^{-3} \mathrm{sec}^{-1}\right)$ always fall in the merging region with the single equilibration mechanism. This likely explains the almost total absence of studies where two jumps in second order thermodynamic properties are found. Nonetheless, it is possible to speculate that there exists a certain class of glass formers exhibiting the splitting (merging) position shifted to time scales short enough for standard calorimetric experiments to show two such jumps in the heat capacity. This could be the case of a siloxane based molecular glass former [14] and PS thin films [15] actually exhibiting two jumps in second order thermodynamic properties, as previously discussed. Considering the unknown nature of the molecular mechanism responsible for the first decay in the enthalpy, whether this corresponds to a kinetic transition cannot be answered at present, although a glass-glass transition may be hypothesized. Further investigation is required to clarify this point.

In conclusion, by means of DSC, we have shown that at temperatures substantially below $T_{g}$, enthalpy recovery in several glass-forming polymers proceeds via a two-step process. The faster one allows partial recovery of the enthalpy, which also depends on the molecular weight, whereas complete recovery, i.e., that expected from extrapolation from the melt, is achieved after the slower process is completed. These results point toward a splitting (merging) scenario and insinuate the presence of two time 
scales for glass equilibration, associated with two different equilibration mechanisms. Analysis of the temperature dependence of the two equilibration times indicates that the faster decay exhibits relatively low activation energy, whereas the slower is adequately described by the same VFT law employed to fit the $\alpha$ process.

The authors acknowledge the University of the Basque Country and Basque Country Government [Reference No. IT-654-13 (G. V.)], Departamento Educación, Universidades e investigación, and the Spanish Government (Grant No. MAT2012-31088) for their financial support.

[1] P. G. Debenedetti, Metastable Liquids: Concepts and Principles (Princeton University, Princeton, NJ, 1996).

[2] A. J. Kovacs, Fortschr. Hochpolym. Forsch. 3, 394 (1964).

[3] L.C.E. Struik, Physical Aging in Amorphous Polymers and Other Materials (Technische Hogeschool Delft, Delft, 1977).

[4] I. M. Hodge, J. Non-Cryst. Solids 169, 211 (1994).

[5] E. Donth, J. Korus, E. Hempel, and M. Beiner, Thermochim. Acta 304-305, 239 (1997).

[6] L. Wang, V. Velikov, and C. Angell, J. Chem. Phys. 117, 10184 (2002).

[7] T. Hecksher, N. B. Olsen, K. Niss, and J. C. Dyre, J. Chem. Phys. 133, 174514 (2010).

[8] H. Vogel, Phys. Z. 22, 645 (1921).

[9] G. S. Fulcher, J. Am. Ceram. Soc. 8, 339 (1925).

[10] G. Tammann and W. Hesse, Z. Anorg. Allg. Chem. 156, 245 (1926).

[11] W. Kauzmann, Chem. Rev. 43, 219 (1948).

[12] D. Cangialosi, A. Alegria, and J. Colmenero, Europhys. Lett. 70, 614 (2005).

[13] C. A. Angell, J. Res. Natl. Inst. Stand. Technol. 102, 171 (1997).

[14] H. Fujimori, M. Mizukami, and M. Oguni, J. Non-Cryst. Solids 204, 38 (1996).

[15] J.E. Pye and C. B. Roth, Phys. Rev. Lett. 107, 235701 (2011).

[16] R. Nozaki and S. Mashimo, J. Chem. Phys. 87, 2271 (1987).

[17] P. A. O'Connell and G. B. McKenna, J. Chem. Phys. 110, 11054 (1999).

[18] S. L. Simon, J. W. Sobieski, and D. J. Plazek, Polymer 42, 2555 (2001).

[19] C. T. Thurau and M. D. Ediger, J. Chem. Phys. 118, 1996 (2003).

[20] D. Cangialosi, M. Wubbenhorst, H. Schut, A. van Veen, and S. J. Picken, Phys. Rev. B 69, 134206 (2004).

[21] T. Hecksher, A. I. Nielsen, N. B. Olsen, and J.C. Dyre, Nat. Phys. 4, 737 (2008).
[22] V.M. Boucher, D. Cangialosi, A. Alegria, and J. Colmenero, Macromolecules 44, 8333 (2011).

[23] B. Ruta, G. Baldi, G. Monaco, and Y. Chushkin, J. Chem. Phys. 138, 054508 (2013).

[24] B. Ruta, Y. Chushkin, G. Monaco, L. Cipelletti, E. Pineda, P. Bruna, V. M. Giordano, and M. Gonzalez-Silveira, Phys. Rev. Lett. 109, 165701 (2012).

[25] I. Avramov and A. Milchev, J. Non-Cryst. Solids 104, 253 (1988).

[26] K. Chen and K. S. Schweizer, Phys. Rev. Lett. 98, 167802 (2007).

[27] Y. S. Elmatad, D. Chandler, and J. P. Garrahan, J. Phys. Chem. B 113, 5563 (2009).

[28] J. C. Mauro, Y. Yue, A. J. Ellison, P. K. Gupta, and D. C. Allan, Proc. Natl. Acad. Sci. U.S.A. 106, 19780 (2009).

[29] R. W. Hall and P. G. Wolynes, J. Chem. Phys. 86, 2943 (1987).

[30] J. Bouchaud and G. Biroli, J. Chem. Phys. 121, 7347 (2004).

[31] C. Bauwens-Crowet and J. Bauwens, Polymer 27, 709 (1986).

[32] A. Brunacci, J.M.G. Cowie, R. Ferguson, and I. J. McEwen, Polymer 38, 3263 (1997).

[33] J. M. M. Duenas, A. V. Garayo, F. R. Colomer, J.M. Estelles, J. L. G. Ribelles, and M. M. Pradas, J. Polym. Sci. B 35, 2201 (1997).

[34] L. Andreozzi, M. Faetti, M. Giordano, and F. Zulli, Macromolecules 38, 6056 (2005).

[35] E. McGonigle, J. Cowie, V. Arrighi, and R. Pethrick, J. Mater. Sci. 40, 1869 (2005).

[36] Y.P. Koh and S.L. Simon, Macromolecules 46, 5815 (2013).

[37] R. C. Welch, J. R. Smith, M. Potuzak, X. Guo, B. F. Bowden, T. J. Kiczenski, D. C. Allan, E. A. King, A.J. Ellison, and J.C. Mauro, Phys. Rev. Lett. 110, 265901 (2013).

[38] V.M. Boucher, D. Cangialosi, A. Alegría, and J. Colmenero, Macromolecules 43, 7594 (2010).

[39] H. Chen, A. Inoue, and T. Masumoto, J. Mater. Sci. 20, 2417 (1985)

[40] R. Golovchak, A. Kozdras, V. Balitska, and O. Shpotyuk, J. Phys. Condens. Matter 24, 505106 (2012).

[41] R. Wimberger Friedl and J. G. de Bruin, Macromolecules 29, 4992 (1996).

[42] C. G. Robertson and G. L. Wilkes, Macromolecules 33, 3954 (2000)

[43] S. Napolitano and M. Wuebbenhorst, Polymer 51, 5309 (2010).

[44] M. Chowdhury, P. Freyberg, F. Ziebert, A. C.-M. Yang, U. Steiner, and G. Reiter, Phys. Rev. Lett. 109, 136102 (2012).

[45] J. Zhao, S. L. Simon, and G. B. McKenna, Nat. Commun. 4, 1783 (2013). 